\title{
ÉPOCA DE MATURAÇÃO, CARACTERIZAÇÃO FÍSICA E QUÍMICA DE CULTIVARES E SELEÇÕES DE CASTANHEIRO ${ }^{1}$
}

\author{
RAFAEL PIO ${ }^{2}$, SILVANA CATARINA SALES BUENO ${ }^{3}$, LUANA APARECIDA CASTILHO MARO ${ }^{4}$, \\ JOÃO PEDRO SALES BUENO ${ }^{5}$, CYNTHIA NATALLY DE ASSIS ${ }^{5}$
}

RESUMO - Nove cultivares ('Taishowase', 'Tiodowase', 'Tamatsukuri', 'Isumo', 'Okuni', 'Moriwase', 'Kinshu', 'Senri' e 'Ibuki') e duas seleções ('KM-2' e 'KM-1') de castanheiro híbrido (Castanea crenata $\mathrm{x}$ Castanea sp.) foram analisadas em São Bento do Sapucaí-SP. Avaliaram-se o início e o término da colheita, a deiscência do fruto e a deiscência, as propriedades físicas, tais como dimensões e massas dos frutos e castanhas, além do formato das castanhas e a poliembrionia, e ainda as propriedades químicas e a composição mineral. Os dados apresentados indicam que as cultivares e seleções diferem quanto à deiscência dos frutos. A colheita das castanhas concentra-se entre a primeira quinzena de novembro e a segunda quinzena de abril. Algumas cultivares apresentaram reduzido número de castanhas dentro da cápsula, o que indica falta de sincronia no período de floração. Há diferença na constituição química das castanhas entre as cultivares e seleções. As castanhas analisadas possuem alta quantidade de proteínas e açúcares totais, baixa quantidade de sódio e extrato etérico, indicando que o amido é a principal substância de reservas das castanhas, das cultivares e seleções analisadas.

Termos para Indexação: Castanea crenata x Castanea sp., florescimento, produção.

\section{MATURATION SEASON, PHYSICS AND CHEMICAL CHARACTERIZATION OF CHESTNUT CULTIVARS AND SELECTIONS}

\begin{abstract}
Nine cultivars ('Taishowase', 'Tiodowase', 'Tamatsukuri', 'Isumo', 'Okuni', 'Moriwase', 'Kinshu', 'Senri' and 'Ibuki') and two selections ('KM-2' and 'KM-1') of the hybrid chestnut (Castanea crenata x Castanea sp.) were analyzed in São Bento do Sapucai-SP. The beginning and the end of the harvest season, the fruit dehiscence and the dehiscence, the physical properties such as dimensions and masses of fruit and nuts, the format of the nuts and polyembryony, and even the chemical and mineral composition were evaluated. The data presented indicate that the cultivars and the selections differ in the dehiscence of the fruits. The harvest of the chestnuts concentrated between the first half of November and the second half of April. Some cultivars showed reduced number of nuts in the hedgehog, which indicates a lack of synchrony in the flowering period. There is difference in the chemical composition of nuts among cultivars and selections. The nuts analyzed have high amount of protein and sugars, low amount of sodium and ethereal extract, indicating that starch is the main substance of chestnuts reserves, in cultivars and selections analyzed. Index terms: Castanea crenata $\mathrm{x}$ Castanea sp., flowering, production.
\end{abstract}

\footnotetext{
'(Trabalho 254-13). Recebido em: 01-08-2013. Aceito para publicação 04-06-2014.

${ }^{2}$ Engenheiro Agrônomo, D.Sc., Professor do Depto. de Agricultura, Universidade Federal de Lavras - UFLA, Dep. de Agricultura, Caixa Postal 3037, 37200-000, Lavras-MG. E-mail: rafaelpio@dag.ufla.br

${ }^{3}$ Engenheira Agrônoma, D.Sc., Secretaria da Agricultura e Abastecimento do Estado de São Paulo, Coordenadoria de Assistência Técnica Integral - CATI, Núcleo de Produção de Mudas de São Bento do Sapucaí, 12490-000, São Bento do Sapucaí-SP. E-mail: scsbueno@cati.sp.gov.br

${ }^{4}$ Engenheira Agrônoma, Bolsista Pós-Doutorado do Programa de Pós-graduação em Fitotecnia, Universidade Federal de Lavras UFLA, Dep. de Agricultura, Caixa Postal 3037, 37200-000, Lavras-MG. E-mail: luana_maro@yahoo.com.br

${ }^{5}$ Estudante do curso de graduação em Agronomia, Universidade Federal de Lavras - UFLA, Dep. de Agricultura, Caixa Postal 3037, 37200-000, Lavras-MG. E-mails: jpbiosales@hotmail.com; cynthianatally@hotmail.com
} 


\section{INTRODUÇÃO}

O castanheiro pertence à família Fagaceae, gênero Castanea, produz frutos tipo cápsula, conhecidos como ouriços que alojam, em seu interior, castanhas de alto valor comercial (CONEDERA et al., 2004). O gênero Castanea apresenta sete espécies das quais se destacam C. sativa Miller, $C$. crenata Siebold \& Zucc., C. molissima Blume e $C$. dentata (Marsh.) Borkh. Tais espécies receberam denominações de acordo com o local de origem e são conhecidas, respectivamente, por castanhaportuguesa (Portugal), castanha- japonesa (Japão e Coreia do Sul), castanha-chinesa (China) e castanhaamericana (América do Norte) (BUENO et al., 2009).

A Coordenadoria de Assistência Técnica Integral (CATI) possui uma coleção com nove cultivares e duas seleções, todas híbridas entre Castanea crenata $\mathrm{x}$ Castanea $\mathrm{sp}$. (YAMANISHI et al., 2010). No entanto, desconhece-se o desempenho produtivo, bem como a composição química e os atributos físicos dos frutos dessas cultivares e seleções, o que tem limitado a recomendação das cultivares para plantios comerciais.

As castanhas contêm pouca gordura, proteína de alta qualidade (3\% da massa total) e são livres de glúten. Também possuem quantidade razoável de vitamina $\mathrm{C}$ e potássio, baixo teor de sódio e cerca de $45 \%$ de amido em suas castanhas, além de conter ácidos graxos em sua composição, que pode variar entre as espécies e até mesmo entre as cultivares do castanheiro (DESMAISON et al., 2001;YAMANISHI et al., 2010). O mesmo já foi relatado em oliveira (OLIVEIRA et al., 2012a; 2012b) e nogueira-macadâmia (MARO et al., 2012), nas quais os autores encontraram diferenças expressivas entre os teores de ácidos graxos essenciais e não essenciais entre as cultivares dessas frutíferas, bem como as características químicas dos frutos entre as cultivares.

Diante do exposto, o presente trabalho teve como objetivo realizar a caracterização da época de maturação, física e química de diferentes cultivares e seleções de castanheiro.

\section{MATERIAL E MÉTODOS}

Mudas enxertadas no porta-enxerto 'Tamatsukuri', de nove cultivares ('Taishowase', 'Tiodowase', 'Tamatsukuri', 'Isumo', 'Okuni', 'Moriwase', 'Kinshu', 'Senri' e 'Ibuki') e duas seleções ('KM-2' e 'KM-1') de castanheiro híbrido (Castanea crenata x Castanea sp.) foram levadas a campo em 1980, no Núcleo de Produção de Mudas da CATI (Coordenadoria de Assistência Técnica Integral) de São Bento do Sapucaí-SP. O local situa-se a uma altitude de mil metros e, de acordo com a classificação de Köppen, apresenta clima
Cwb (subtropical de altitude), com precipitação média anual entre 1.600 e $1.800 \mathrm{~mm}$, temperatura média anual entre $12^{\circ}$ e $18^{\circ} \mathrm{C}$. O solo do local está classificado como LVA (Latossolo VermelhoAmarelo) que se caracteriza por ser um solo profundo, com fertilidade moderada e boa capacidade de retenção de água.

Foram demarcadas quatro plantas de cada cultivar ou seleção para as avaliações, distribuídas ao acaso na área de plantio, em julho de 2012. Foram demarcados o início e o término da colheita, bem como a deiscência, ou seja, se as cápsulas permaneciam fixas nas plantas apenas liberando as castanhas ou se desprendiam das plantas.

Durante a colheita, de cada quatro plantas, que representaram um bloco, colheram-se 20 cápsulas maduras e aparentemente sadias, de forma aleatória, no período da manhã, para as análises físicas e químicas. Assim, o delineamento utilizado nas avaliações foi em blocos ao acaso, com 11 tratamentos (cultivares e seleções de castanheiro), contendo quatro blocos e 20 frutos por parcela. Os frutos foram transportados em caixas abertas para o laboratório de pomologia do Depto. de Agricultura, localizado no pomar didático da Universidade Federal de Lavras (UFLA), Lavras-MG.

As variáveis físicas avaliadas foram: massa total da cápsula contento as castanhas, diâmetro longitudinal (DL) e diâmetro transversal (DT) da cápsula e castanha, espessura da porção mediana das castanhas, relação DL/DT, classificação do formato das castanhas [cujos resultados indicam castanhas chatas (DL/DT $<1)$, levemente redondas $(\mathrm{DL} / \mathrm{DT}=1)$ ou alongadas $(\mathrm{DL} / \mathrm{DT}>1)$ ], número total de castanhas por cápsula, número médio de castanhas polinizadas e não polinizadas, massa total e média das castanhas por cápsula, porcentagem de castanhas com rachaduras, porcentagem de castanhas monoembriônicas e poliembriônicas (compartimentação), ou seja, com dois ou mais embriões. Todas essas análises, quando necessárias, foram realizadas com auxílio de paquímetro digital e balança de precisão, de acordo com Penoni et al. (2011).

Posteriormente às análises físicas, 40 castanhas por bloco de cada cultivar ou seleção foram descascadas e utilizadas para a avaliação das seguintes características químicas: acidez total titulável (trituração em politron, com dois gramas de castanhas transferidas para erlenmeyers, completando-se o volume para $100 \mathrm{~mL}$ com água destilada, adicionada de três gotas de indicador fenolftaleína a $1 \%$, sob agitação, com solução de $\mathrm{NaOH} 0,01 \mathrm{~N}$, padronizada com biftalato de potássio e resultados expressos em $\mathrm{mg}$ de ácido cítrico por $100 \mathrm{~g}$ de castanha), sólidos solúveis totais (amostra triturada em politron e realizadas as leituras por amostra com o auxílio de um refratômetro digital, com leitura expressa em ${ }^{\circ}$ Brix), vitamina 
C (conteúdo de ácido ascórbico determinado pelo método colorimétrico), açúcares totais e redutores, umidade, cinzas, extrato etéreo, proteína e fibra bruta, além da composição mineral, sendo o nitrogênio pelo método semimicro-Kjeldahl, fósforo e boro por métodos colorimétricos, enxofre por turbidimetria, potássio por fotometria de chama e emissão, e cálcio, magnésio, cobre, ferro, manganês e zinco pelo método de espectrofotometria de absorção atômica.

Os dados das análises químicas e físicas foram submetidos à análise de variância, e as médias foram agrupadas pelo teste de Scott-Knott ao nível de 5\% de probabilidade. Para a variável época de maturação, procedeu-se à análise descritiva dos dados.

\section{RESULTADOS E DISCUSSÃO}

Nas condições climáticas da região de São Bento do Sapucaí-SP, a maioria das cultivares e seleções atingem o ponto de maturação das castanhas em novembro, à exceção da cultivar Tamatsukuri, considerada uma cultivar precoce, cuja colheita se iniciou na segunda quinzena de outubro, 'Kinshu' e KM-1', com colheitas iniciando-se em janeiro e 'Senri', considerada tardia, com época de produção iniciada somente em fevereiro (Tabela 1). De modo geral, a época de produção de castanhas inicia-se na segunda quinzena de outubro e estende-se até o final do mês de abril. Porém, a maior época de consumo de castanhas no Brasil é no Natal, época em que se obtém os melhores preços e, consequentemente, no valor das castanhas (YAMANISHI et al., 2010). Nesse sentido, as cultivares e seleções 'Kinshu', KM1' e 'Senri' seriam de menor interesse.

Quanto à deiscência, foram observadas três formas, sendo elas: castanhas soltam-se e cápsula permanece na planta; cápsula cai aberta ou fechada, no caso ambas com as castanhas dentro da cápsula. Segundo Yamanishi et al. (2010), as castanhas contêm alta quantidade de amido e umidade, o que pode ocasionar a incidência de doenças, quando as castanhas ficam em contato com o solo. No entanto, uma opção seria o uso de redes ou telas, colocadas $50 \mathrm{~cm}$ acima do solo, o que facilitaria a colheita e, nesse caso, as cultivares Taishowase e Tamatsukuri, e as seleções KM-2 e KM-1 proporcionam maior facilitade na operação da colheita, já que elas se desprendem com a deiscência do fruto, que fica retido na planta (Tabela 1).

Quanto à biometria dos frutos, a cultivar Tamatsukuri apresentou maior massa total da cápsula, porém não houve destaque quanto aos diâmetros (Tabela 2). Apesar da diferença estatística, o número médio de castanhas por cápsula foi de três, porém as cultivares Taishowase, Tiodowase, Kinshu e a seleção KM-2 apresentaram maiores quantidades de flores polinizadas, ao passo que Isumo, Okuni, Moriwase, Senri e Ibuki apresentaram maiores números de flores não polinizadas, que não se desenvolvem e permanecem inviáveis para o consumo (Tabela 2). No caso específico da 'Senri', por ser mais tardia que as demais, possívelmente na época de floração não houve outra cultivar em florescimento, o que resultou em baixa quantidade de castanhas polinizadas. Isso refletiu na massa média das castanhas polinizadas, pois, como houve praticamente uma única castanha dentro da cápsula da cultivar Senri, essa desenvolveu-se mais e, consequentemente, apresentou a maior massa (21,18 g) (Tabela 2). Já as cultivares Taishowase e Moriwase, e a seleção KM-2 apresentaram castanhas de menores massa $(9,52 ; 10,45$ e 10,14 g, respectivamente). Pereira-Lorenzo et al. (2006) também constataram grande diferença entre as massas das castanhas, comparando várias cultivares na Espanha, encontrando desde castanhas com 6,45 $\mathrm{g}$ até castanhas com massa média de 22,72 g.

Quanto às dimensões das castanhas, não houve diferença entre os diâmetros e a espessura; no entanto, constatou-se diferença estatística na relação diâmetro longitudinal e transversal (Tabela 3). Assim, as cultivares Isumo e Ibuki apresentaram relação bem menor que 1,0 , sendo classificadas como chatas; já 'Moriwase', 'Kinshu' e 'KM-1', relação maior que 1,0, classificadas como alongadas, e as demais, como redondas (Tabela 3). As cultivares Moriwase e Senri registraram maior porcentagem de castanhas rachadas, e a 'Taishowase' e 'Kinshu' maior porcentagem de castanhas monoembriônicas (acima de 92\%), característica importante quanto ao processamento, na produção do marrom- glacê, já que as castanhas devem permanecer inteiras e intactas.

De modo geral, as castanhas de todas as cultivares e seleções apresentaram baixa acidez, principalmente 'Kinshu', 'KM-2' e 'Senri', sendo essas ainda que registraram os maiores teores de sólidos solúveis totais (Tabela 4). Dentre essas três, a 'KM-2' apresentou a maior quantidade de açúcares totais (559,01 mg em $100 \mathrm{~g}$ de castanha). Esse valor está bem acima dos registrados por Pereira-Lorenzo et al. (2006), em castanhas (Castanea sativa) oriundas da Espanha. Não houve diferença entre as quantidades de açúcares redutores nas castanhas entre as cultivares e seleções, apesar de os valores estarem acima das quantidades encontradas por Ertürk et al. (2006). Também não houve diferença entre as quantidades de vitamina $\mathrm{C}$, apesar de as castanhas não serem uma boa fonte dessa vitamina, como outras frutas, a exemplo da framboesa (MARO et al., 2013).

As castanhas apresentaram alta umidade, apesar de os valores serem ligeiramente menores que os relatados por Pereira-Lorenzo et al. (2006). As cultivares Taishowase, Tamatsukuri, Kinshu e a seleção KM-2 apresentaram maiores teores de cinzas (Tabela 4), com valores maiores que os obtidos por Ertürk et al. (2006). A quantidade de extrato etéreo 
registrado nas castanhas das cultivares e seleções estudadas estão em níveis baixos, estando de acordo com o relatado por Demiatel et al. (2001), que citam que a principal substância de reserva das castanhas é o amido, representado pelas quantidades de açúcares totais. Outras castanhas, como o caso da macadâmia, contêm teores expressivs de extrato etéreo, sendo o óleo a principal substâncias de reservas das macadâmias (MARO et al., 2012). Não houve diferença nas quantidades de fibra bruta entre as cultivares e seleções de castanheiro. 'Tiodowase',
'Tamatsukuri', 'Okuni' e 'Moriwase' apresentaram maiores quantidades de proteínas (Tabela 4), com valores bem superiores aos relatados por PereiraLorenzo et al. (2006) e Ertürk et al. (2006).

Para as quantidades de minerais, a cultivar Tiodowase, no geral, destacou-se entre as demais para as quantidades de macronutrientes, e as cultivares Okuni e Moriwase para as quantidades de micronutrientes, principalmente a 'Moriwase', pela reduzida quantidade de sódio (Tabela 5).

TABELA 1 - Deiscência, início e término da colheita de diferentes cultivares de castanheiro. UFLA, Lavras, 2013.

\begin{tabular}{|c|c|c|c|}
\hline Cultivares & Deiscência & Início da colheita & Término da colheita \\
\hline Taishowase & Castanhas se soltam e cápsula permanece na planta & $1^{\mathrm{a}}$ quinzena nov. & $2^{\circ}$ quinzena fev. \\
\hline Tiodo & astanhas & ena nov. & a dez. \\
\hline Tamatsukuri & Castanhas se soltam e cápsula permanece na planta & na out. & $1^{\circ}$ quinzena dez. \\
\hline Isumo & tro & la nov. & a dez. \\
\hline $\mathrm{KM}-2$ & na planta & ena nov. & $2^{\circ}$ quinzena dez. \\
\hline Okuni & i fechada com as castanhas dentro & $1^{\mathrm{a}}$ quinzena nov. & $2^{\circ}$ quinzena dez. \\
\hline Morioase & Cápsula cai fechada com as castanhas dentro & $2^{\mathrm{a}}$ quinzena nov. & $2^{\circ}$ quinzena dez. \\
\hline hu & has dentro & $1^{\mathrm{a}} \mathrm{q}$ & a fev. \\
\hline $\mathrm{C}_{\mathrm{g}}$ & astanhas dentro & a fev. & abr. \\
\hline KM - 1 & soltam e cáps & jan. & $2^{\circ}$ quinzena fev. \\
\hline Ibuki & Cápsula cai fechada com as castanhas dentro & $2^{\mathrm{a}}$ quinzena nov. & $2^{\circ}$ quinzena dez. \\
\hline
\end{tabular}

TABELA 2 - Massa total, diâmetro longitudinal e transversal da cápsula, número total de castanhas, número de castanhas polinizadas e não polinizadas, massa total e média de castanhas polinizadas em diferentes cultivares de castanheiro. UFLA, Lavras, 2013.

\begin{tabular}{lcccc}
\hline Cultivares & $\begin{array}{c}\text { Massa total } \\
\text { cápsula }(\mathrm{g})\end{array}$ & $\begin{array}{c}\text { Diâmetro longitudinal } \\
\text { cápsula }(\mathrm{mm})\end{array}$ & $\begin{array}{c}\text { Diâmetro transversal } \\
\text { cápsula }(\mathrm{mm})\end{array}$ & $\mathrm{N}^{\circ}$ total castanhas \\
Taishowase & $13,74 \mathrm{e}$ & $45,53 \mathrm{f}$ & $62,64 \mathrm{c}$ & $2,95 \mathrm{c}$ \\
Tiodowase & $30,58 \mathrm{c}$ & $65,10 \mathrm{c}$ & $87,81 \mathrm{a}$ & $3,20 \mathrm{~b}$ \\
Tamatsukuri & $48,09 \mathrm{a}$ & $59,91 \mathrm{~d}$ & $82,68 \mathrm{~b}$ & $3,17 \mathrm{~b}$ \\
Isumo & $41,37 \mathrm{~b}$ & $65,33 \mathrm{c}$ & $78,23 \mathrm{~b}$ & $3,00 \mathrm{c}$ \\
KM -2 & $17,39 \mathrm{e}$ & $53,88 \mathrm{e}$ & $67,82 \mathrm{c}$ & $3,01 \mathrm{c}$ \\
Okuni & $33,86 \mathrm{c}$ & $68,66 \mathrm{~b}$ & $81,36 \mathrm{~b}$ & $3,00 \mathrm{c}$ \\
Morioase & $41,77 \mathrm{~b}$ & $74,74 \mathrm{a}$ & $87,98 \mathrm{a}$ & $3,65 \mathrm{a}$ \\
Kinshu & $20,67 \mathrm{~d}$ & $63,25 \mathrm{c}$ & $81,31 \mathrm{~b}$ & $3,00 \mathrm{c}$ \\
Senri & $23,66 \mathrm{~d}$ & $73,39 \mathrm{a}$ & $85,35 \mathrm{a}$ & $3,20 \mathrm{~b}$ \\
KM - 1 & $33,45 \mathrm{c}$ & $69,69 \mathrm{~b}$ & $88,72 \mathrm{a}$ & $3,00 \mathrm{c}$ \\
Ibuki & $38,40 \mathrm{~b}$ & $54,78 \mathrm{e}$ & $66,04 \mathrm{c}$ & $2,97 \mathrm{c}$ \\
CV (\%) & 10,87 & 3,95 & 4,34 & 3,96 \\
\hline & $\mathrm{N}^{\circ}$ castanhas & $\mathrm{N}^{0}$ castanhas não & Massa total castanhas & Massa média castanhas \\
Taishowase & polinizadas & polinizadas & polinizadas $(\mathrm{g})$ & polinizadas $(\mathrm{g})$ \\
Tiodowase & $2,72 \mathrm{a}$ & $0,23 \mathrm{c}$ & $25,37 \mathrm{c}$ & $9,52 \mathrm{c}$ \\
Tamatsukuri & $2,95 \mathrm{a}$ & $0,25 \mathrm{c}$ & $43,78 \mathrm{a}$ & $15,20 \mathrm{~b}$ \\
Isumo & $2,10 \mathrm{~b}$ & $1,07 \mathrm{~b}$ & $28,51 \mathrm{c}$ & $12,89 \mathrm{~b}$ \\
KM -2 & $1,15 \mathrm{c}$ & $1,85 \mathrm{a}$ & $19,75 \mathrm{~d}$ & $13,25 \mathrm{~b}$ \\
Okuni & $2,67 \mathrm{a}$ & $0,34 \mathrm{c}$ & $27,19 \mathrm{c}$ & $10,14 \mathrm{c}$ \\
Morioase & $1,02 \mathrm{c}$ & $1,98 \mathrm{a}$ & $17,83 \mathrm{~d}$ & $13,12 \mathrm{~b}$ \\
Kinshu & $2,15 \mathrm{~b}$ & $1,50 \mathrm{a}$ & $21,97 \mathrm{~d}$ & $10,45 \mathrm{c}$ \\
Senri & $2,47 \mathrm{a}$ & $0,53 \mathrm{c}$ & $37,13 \mathrm{~b}$ & $14,66 \mathrm{~b}$ \\
KM - 1 & $1,30 \mathrm{c}$ & $1,90 \mathrm{a}$ & $28,79 \mathrm{c}$ & $21,18 \mathrm{a}$ \\
Ibuki & $2,20 \mathrm{~b}$ & $0,80 \mathrm{~b}$ & $33,39 \mathrm{~b}$ & $15,28 \mathrm{~b}$ \\
CV (\%) & $1,22 \mathrm{c}$ & $1,75 \mathrm{a}$ & $20,92 \mathrm{~d}$ & $14,75 \mathrm{~b}$ \\
\hline
\end{tabular}

*Médias seguidas pela mesma letra na coluna não diferem entre si, pelo teste de Scott- Knott $(\mathrm{P} \leq 0,05)$. 
TABELA3 - Diâmetro longitudinal, transversal, espessura, relação diâmetro longitudinal e transversal, e formato das castanhas, porcentagem de castanhas rachadas, porcentagem de castanhas monoembriônicas e poliembriônicas em diferentes cultivares de castanheiro. UFLA, Lavras, 2013.

\begin{tabular}{|c|c|c|c|c|}
\hline Cultivares & $\begin{array}{l}\text { Diâmetro longitudinal } \\
\text { castanha }(\mathrm{DL}, \mathrm{mm})\end{array}$ & $\begin{array}{l}\text { Diâmetro transversal } \\
\text { castanha (DT, mm) }\end{array}$ & Espessura (mm) & $\mathrm{DL} / \mathrm{DT}$ \\
\hline 'Taishowase' & $27,93^{\text {ns }}$ & $28,96^{\mathrm{ns}}$ & $39,48^{\mathrm{ns}}$ & $0,97 \mathrm{~b}$ \\
\hline 'Tiodowase' & 33,61 & 34,22 & 42,06 & $0,99 \mathrm{~b}$ \\
\hline 'Tamatsukuri' & 31,54 & 32,03 & 39,06 & $0,94 \mathrm{~b}$ \\
\hline 'Isumo’' & 26,54 & 26,39 & 30,70 & $0,75 \mathrm{c}$ \\
\hline 'KM - 2' & 31,01 & 30,58 & 40,24 & $1,02 \mathrm{~b}$ \\
\hline ‘Okuni’ & 36,59 & 31,72 & 39,75 & $1,09 \mathrm{~b}$ \\
\hline 'Morioase' & 32,79 & 18,87 & 26,14 & $1,67 \mathrm{a}$ \\
\hline 'Kinshu' & 32,09 & 20,46 & 32,99 & $1,53 \mathrm{a}$ \\
\hline ‘Senri’ & 32,89 & 24,63 & 35,33 & $1,19 \mathrm{~b}$ \\
\hline ' $\mathrm{KM}-1$ ' & 37,17 & 21,42 & 36,69 & $1,76 \mathrm{a}$ \\
\hline 'Ibuki' & 28,50 & 29,35 & 35,35 & $0,78 \mathrm{c}$ \\
\hline $\mathrm{CV}(\%)$ & 22,10 & 27,14 & 17,50 & 12,30 \\
\hline \multicolumn{3}{|c|}{ Formato das castanhas $\%$ de castanhas rachadas } & $\begin{array}{l}\text { \% de castanhas } \\
\text { monoembriônicas }\end{array}$ & $\begin{array}{c}\text { \% de castanhas } \\
\text { poliembriônicas }\end{array}$ \\
\hline 'Taishowase' & redondas & $0,82 \mathrm{~b}$ & $97,50 \mathrm{a}$ & $2,50 \mathrm{~d}$ \\
\hline ‘Tiodowase' & redondas & $1,87 \mathrm{~b}$ & $44,15 \mathrm{~b}$ & $55,85 \mathrm{a}$ \\
\hline 'Tamatsukuri' & redondas & $6,65 \mathrm{~b}$ & $50,20 \mathrm{~b}$ & $44,80 \mathrm{~b}$ \\
\hline 'Isumo’' & chatas & $7,50 \mathrm{~b}$ & $54,17 \mathrm{~b}$ & $20,82 \mathrm{c}$ \\
\hline 'KM - 2' & redondas & $6,67 \mathrm{~b}$ & $60,90 \mathrm{~b}$ & $39,10 \mathrm{~b}$ \\
\hline 'Okuni' & redondas & $10,00 \mathrm{~b}$ & $39,17 \mathrm{~b}$ & $35,82 \mathrm{~b}$ \\
\hline 'Morioase' & alongadas & $23,55 \mathrm{a}$ & $33,70 \mathrm{~b}$ & $61,30 \mathrm{a}$ \\
\hline ‘Kinshu’ & alongadas & $4,55 \mathrm{~b}$ & $92,52 \mathrm{a}$ & $4,97 \mathrm{~d}$ \\
\hline 'Senri' & redondas & $32,50 \mathrm{a}$ & $59,57 \mathrm{~b}$ & $27,92 \mathrm{c}$ \\
\hline ' $\mathrm{KM}-1$ ' & alongadas & $6,67 \mathrm{~b}$ & $57,23 \mathrm{~b}$ & $42,77 \mathrm{~b}$ \\
\hline ‘Ibuki’ & chatas & $10,00 \mathrm{~b}$ & $54,17 \mathrm{~b}$ & $25,82 \mathrm{c}$ \\
\hline CV (\%) & - & 66,79 & 24,45 & 37,54 \\
\hline
\end{tabular}

* Médias seguidas pela mesma letra na coluna não diferem entre si, pelo teste de Scott- Knott $(\mathrm{P} \leq 0,05)$.

TABELA 4 - Acidez total titulável, teores de sólidos solúveis totais, vitamina $C$, açúcares totais e redutores, de umidade, cinzas, extrato etéreo, proteínas e fibra bruta em castanhas de diferentes cultivares de castanheiro. UFLA, Lavras, 2013.

\begin{tabular}{lccccc}
\hline Cultivares & $\begin{array}{c}\text { Acidez total } \\
\text { titulável } \\
\%\end{array}$ & $\begin{array}{c}\text { Sólidos solúveis } \\
\text { totais } \\
\text { '0 }\end{array}$ & Vitamina C & Açúcares totais & Açúcares redutores \\
'Taishowase' & $0,04 \mathrm{~d}$ & $7,05 \mathrm{~b}$ & $10,44^{\mathrm{ns}}$ & $242,89 \mathrm{c}$ & $27,82^{\mathrm{ns}}$ \\
'Tiodowase' & $0,07 \mathrm{~d}$ & $9,37 \mathrm{~b}$ & 12,60 & $237,45 \mathrm{c}$ & 31,30 \\
'Tamatsukuri' & $0,15 \mathrm{~b}$ & $9,30 \mathrm{~b}$ & 12,29 & $390,12 \mathrm{~b}$ & 25,74 \\
'Isumo' & $0,17 \mathrm{~b}$ & $9,07 \mathrm{~b}$ & 12,60 & $336,83 \mathrm{c}$ & 26,29 \\
'KM - 2' & $0,13 \mathrm{c}$ & $12,22 \mathrm{a}$ & 10,75 & $559,01 \mathrm{a}$ & 27,91 \\
'Okuni' & $0,15 \mathrm{~b}$ & $8,10 \mathrm{~b}$ & 11,67 & $290,00 \mathrm{c}$ & 23,28 \\
'Morioase' & $0,17 \mathrm{~b}$ & $8,40 \mathrm{~b}$ & 11,88 & $260,80 \mathrm{c}$ & 25,94 \\
'Kinshu' & $0,12 \mathrm{c}$ & $12,37 \mathrm{a}$ & 11,67 & $417,18 \mathrm{~b}$ & 33,88 \\
'Senri' & $0,12 \mathrm{c}$ & $10,80 \mathrm{a}$ & 10,14 & $354,59 \mathrm{c}$ & 25,28 \\
'KM - 1' & $0,14 \mathrm{a}$ & $8,40 \mathrm{~b}$ & 10,44 & $261,07 \mathrm{c}$ & 25,52 \\
'Ibuki' & $0,24 \mathrm{a}$ & $9,52 \mathrm{~b}$ & 13,52 & $354,67 \mathrm{c}$ & 23,37 \\
CV $(\%)$ & 15,96 & 14,39 & 9,85 & 22,22 & 5,23 \\
\hline
\end{tabular}


R. PIO et al.

\begin{tabular}{lccccc}
\hline & Umidade & Cinzas & Extrato etéreo & Proteínas & Fibra bruta \\
'Taishowase' & 48,30 a & $0,64 \mathrm{a}$ & $0,095 \mathrm{~b}$ & $5,37 \mathrm{~d}$ & $2,67 \mathrm{~ns}$ \\
'Tiodowase' & $43,02 \mathrm{~b}$ & $0,44 \mathrm{~b}$ & $0,020 \mathrm{~b}$ & $10,35 \mathrm{a}$ & 2,53 \\
'Tamatsukuri' & $44,33 \mathrm{a}$ & $0,55 \mathrm{a}$ & $0,060 \mathrm{~b}$ & $10,56 \mathrm{a}$ & 2,15 \\
'Isumo' & $38,91 \mathrm{~b}$ & $0,38 \mathrm{~b}$ & $0,082 \mathrm{~b}$ & $9,25 \mathrm{~b}$ & 3,02 \\
'KM - 2' & $49,38 \mathrm{a}$ & $0,60 \mathrm{a}$ & $0,160 \mathrm{a}$ & $7,23 \mathrm{c}$ & 2,99 \\
'Okuni' & $40,40 \mathrm{~b}$ & $0,43 \mathrm{~b}$ & $0,054 \mathrm{~b}$ & $11,06 \mathrm{a}$ & 2,78 \\
'Morioase' & $46,39 \mathrm{a}$ & $0,46 \mathrm{~b}$ & $0,061 \mathrm{~b}$ & $11,31 \mathrm{a}$ & 2,11 \\
'Kinshu' & $48,85 \mathrm{a}$ & $0,65 \mathrm{a}$ & $0,043 \mathrm{~b}$ & $7,02 \mathrm{c}$ & 2,78 \\
'Senri' & $38,16 \mathrm{~b}$ & $0,39 \mathrm{~b}$ & $0,068 \mathrm{~b}$ & $7,69 \mathrm{c}$ & 2,24 \\
'KM - 1' & $46,01 \mathrm{a}$ & $0,41 \mathrm{~b}$ & $0,140 \mathrm{a}$ & $7,77 \mathrm{c}$ & 1,98 \\
'Ibuki' & $40,59 \mathrm{~b}$ & $0,36 \mathrm{~b}$ & $0,054 \mathrm{~b}$ & $9,37 \mathrm{~b}$ & 2,08 \\
CV (\%) & 8,45 & 22,37 & 48,70 & 8,45 & 12,03 \\
\hline
\end{tabular}

*Médias seguidas pela mesma letra na coluna não diferem entre si pelo Teste de Scott- Knott $(\mathrm{P} \leq 0,05)$.

TABELA 5 - Teores de macro e micronutrientes: nitrogênio $(\mathrm{N})$, fósforo $(\mathrm{P})$, potássio $(\mathrm{K})$, magnésio $(\mathrm{Mg})$, enxofre $(\mathrm{S})$, sódio $(\mathrm{Na})$, boro $(\mathrm{B})$, cobre $(\mathrm{Cu})$, manganês $(\mathrm{Mn})$, ferro $(\mathrm{Fe})$ e zinco $(\mathrm{Zn}) \mathrm{em}$ castanhas de diferentes cultivares de castanheiro. UFLA, Lavras, 2013.

\begin{tabular}{|c|c|c|c|c|c|c|}
\hline Cultivares & $\mathrm{N}$ & $\mathrm{P}$ & $\mathrm{K}$ & $\mathrm{Ca}$ & $\mathrm{Mg}$ & $\mathrm{S}$ \\
\hline 'Taishowase' & $0,90 \mathrm{~d}$ & $0,13 \mathrm{c}$ & $11,30 \mathrm{a}$ & $0^{\text {ns }}$ & $0,011 \mathrm{a}$ & $0,02 \mathrm{c}$ \\
\hline 'Tiodowase' & $1,66 \mathrm{a}$ & $1,73 \mathrm{a}$ & $11,10 \mathrm{a}$ & 0 & $0,012 \mathrm{a}$ & $0,04 \mathrm{~b}$ \\
\hline Tamatsukuri & $1,69 \mathrm{a}$ & $1,93 \mathrm{a}$ & $9,83 \mathrm{~b}$ & 0 & $0,010 b$ & $0,07 \mathrm{a}$ \\
\hline 'Isumo' & $1,48 \mathrm{~b}$ & $1,70 \mathrm{a}$ & $7,90 \mathrm{c}$ & 0 & $0,090 \mathrm{c}$ & $0,06 \mathrm{a}$ \\
\hline 'KM - 2' & $1,16 \mathrm{c}$ & $0,97 \mathrm{~d}$ & $8,30 \mathrm{c}$ & 0 & $0,011 \mathrm{~b}$ & $0,00 \mathrm{c}$ \\
\hline 'Okuni' & $1,77 \mathrm{a}$ & $1,80 \mathrm{a}$ & $10,03 \mathrm{~b}$ & 0 & $0,010 \mathrm{~b}$ & $0,05 \mathrm{~b}$ \\
\hline 'Morioase' & $1,81 \mathrm{a}$ & $1,80 \mathrm{a}$ & $11,00 \mathrm{a}$ & 0 & $0,010 \mathrm{~b}$ & $0,04 \mathrm{~b}$ \\
\hline 'Kinshu' & $1,12 \mathrm{c}$ & $0,80 \mathrm{~d}$ & $8,70 \mathrm{c}$ & 0 & $0,080 \mathrm{c}$ & $0,00 \mathrm{c}$ \\
\hline 'Senri' & $1,23 \mathrm{c}$ & $1,27 \mathrm{c}$ & $10,20 \mathrm{~b}$ & 0 & $0,011 \mathrm{~b}$ & $0,00 \mathrm{c}$ \\
\hline ' $\mathrm{KM}-1$ ' & $1,24 \mathrm{c}$ & $1,47 \mathrm{~b}$ & $9,33 \mathrm{c}$ & 0 & $0,097 \mathrm{c}$ & $0,03 \mathrm{~b}$ \\
\hline 'Ibuki' & $1,50 \mathrm{~b}$ & $1,43 \mathrm{~b}$ & $8,17 \mathrm{c}$ & 0 & $0,010 \mathrm{~b}$ & $0,04 \mathrm{~b}$ \\
\hline \multirow[t]{2}{*}{ CV $(\%)$} & 10,25 & 15,74 & 7,12 & 0 & 10,29 & 45,66 \\
\hline & $\mathrm{Na}$ & B & $\mathrm{Cu}$ & $\mathrm{Mn}$ & $\mathrm{Fe}$ & $\mathrm{Zn}$ \\
\hline 'Taishowase' & $36,20 \mathrm{c}$ & $13,43 \mathrm{~b}$ & $4,80 \mathrm{~b}$ & $1,53 \mathrm{~ns}$ & $36,23 \mathrm{~b}$ & $8,60 \mathrm{~d}$ \\
\hline 'Tiodowase' & $52,80 \mathrm{~b}$ & $13,80 \mathrm{~b}$ & $6,73 \mathrm{a}$ & 2,17 & $51,81 \mathrm{a}$ & $14,40 \mathrm{~b}$ \\
\hline 'Tamatsukuri' & $36,92 \mathrm{c}$ & $12,60 \mathrm{~b}$ & $5,90 \mathrm{a}$ & 2,70 & $41,17 \mathrm{~b}$ & $20,07 \mathrm{a}$ \\
\hline 'Isumo' & $38,72 \mathrm{c}$ & $12,73 \mathrm{~b}$ & $4,73 \mathrm{~b}$ & 1,97 & $38,60 \mathrm{~b}$ & $15,90 \mathrm{~b}$ \\
\hline$' \mathrm{KM}-2$ ' & $33,12 \mathrm{c}$ & $10,47 \mathrm{c}$ & $4,27 \mathrm{~b}$ & 1,50 & $36,43 \mathrm{~b}$ & $9,33 \mathrm{~d}$ \\
\hline 'Okuni' & $54,95 \mathrm{~b}$ & $17,10 \mathrm{a}$ & $8,23 \mathrm{a}$ & 2,43 & $48,47 \mathrm{a}$ & $19,73 \mathrm{a}$ \\
\hline 'Morioase' & $44,30 \mathrm{c}$ & $16,75 \mathrm{a}$ & $7,10 \mathrm{a}$ & 2,70 & $52,30 \mathrm{a}$ & $14,65 \mathrm{~b}$ \\
\hline 'Kinshu' & $33,82 \mathrm{c}$ & 16,93 a & $4,27 \mathrm{~b}$ & 3,90 & $40,40 \mathrm{~b}$ & $9,67 \mathrm{~d}$ \\
\hline 'Senri' & $72,60 \mathrm{a}$ & $16,43 \mathrm{a}$ & $4,30 \mathrm{~b}$ & 2,73 & $43,20 \mathrm{~b}$ & $12,37 \mathrm{c}$ \\
\hline ' $\mathrm{KM}-1$ ' & $29,80 \mathrm{c}$ & $15,40 \mathrm{a}$ & $6,43 \mathrm{a}$ & 1,23 & $40,32 \mathrm{~b}$ & $13,03 \mathrm{c}$ \\
\hline 'Ibuki' & $46,20 \mathrm{c}$ & $13,03 \mathrm{~b}$ & $6,47 \mathrm{a}$ & 4,73 & $35,40 \mathrm{~b}$ & $15,37 b$ \\
\hline CV (\%) & 21,25 & 9,29 & 17,73 & 2,96 & 13,60 & 18,09 \\
\hline
\end{tabular}

*Médias seguidas pela mesma letra na coluna não diferem entre si, pelo teste de Scott- Knott $(\mathrm{P} \leq 0,05)$. 


\section{CONCLUSÕES}

A colheita das castanhas concentra-se entre a primeira quinzena de novembro e a segunda quinzena de abril, sendo 'Tamatsukuri' a mais precoce e 'Senri' a mais tardia. Algumas cultivares apresentam reduzido número de castanhas dentro da cápsula, porém com castanhas de maiores massas. 'Kinshu' é uma boa opção para o processamento, por apresentar castanhas monoembriônicas, baixa acidez, altor teor de sólidos solúveis e cinzas. Para o consumo ao natural, a cultivar 'KM-2' destacouse, pela alta quantidade de sólidos solúveis, baixa acidez e açúcares totais.

\section{AGRADECIMENTOS}

À Fundação de Amparo à Pesquisa do Estado de Minas Gerais (FAPEMIG) e à Coordenação de Aperfeiçoamento de Pessoal de Nível Superior (CAPES), pelo apoio financeiro na execução deste trabalho.

\section{REFERÊNCIAS}

BUENO, S.C.S.; COUTINHO, E.; MAIA, A.H.; YAMANISHI, O. Grafting compatibility among eleven chestnut cultivars and hybrids. Acta Horticulturae, Leuven, v.844, p.127-131, 2009.

CONEDERA, M.; KREBS, P.; TINNER, W.; PRADELLA, M.; TORRIANI, D. The cultivation of Castanea sativa (Mill.) in Europe, from its origin to its diffusion on a continental scale. Vegetation History and Archaeobotany, Wilhelmshaven, v.13, n.3, p.161-179, 2004.

DEMIATEL, I.M.; OETTERER, M.; WOSIACKI, G. Characterization of chestnut (Castanea sativa, Mill) starch for industrial utilization. Brazilian Archives of Biology and Technology, Curitiba, v.44, n.1, p.69-78, 2001.

DESMAISON, A.M.; MARCHER, M.H.; TIXIER, M. Changes in the free and total amino acid composition of ripening chestnut seeds. Phytochemistry, Pullman, v.23, n.11, p.2.4532.456, 2001.
ERTÜRK, Ü.; MERT, C.; SOYLU, A. Chemical composition of fruits of some important chestnut cultivars. Brazilian Archives of Biology and Technology, Curitiba, v.49, n.2, p.183-188, 2006.

MARO, L.A.C.; PIO, R.; GUEDES, M.N.S.; ABREU, C.M.P.; CURI, P.N. Bioactive compounds, antioxidant activity and mineral composition of fruits of raspberry cultivars grown in subtropical areas in Brazil. Fruits, Montpellier, v.68, n.3, p.1-9, 2013.

MARO, L.A.C.; PIO, R.; PENONI, E.S.; OLIVEIRA, M.C.; PRATES, F.C.; LIMA, L.C.O.; CARDOSO, M.G. Caracterização química e perfil de ácidos graxos em cultivares de nogueiramacadâmia. Ciência Rural, Santa Maria, v.42, n.12, p.2.166-2.171, 2012.

OLIVEIRA, L.F.; OLIVEIRA, A.F.; PIO, R.; ALVES, T.C.; ZAMBON, C.R. Variação na qualidade do azeite em cultivares de oliveira. Bragantia, Campinas, v.71, n.2, p.202-209, 2012 b.

OLIVEIRA, M.C.; RAMOS, J.D.; PIO, R.; CARDOSO, M.G. Características fenológicas e físicas e perfil de ácidos graxos em oliveiras no sul de Minas Gerais. Pesquisa Agropecuária Brasileira, Brasília, v.47, n.1, p.30-35, 2012a.

PENONI, E.S.; PIO, R.; RODRIGUES, F.A.; MARO, L.A.C.; COSTA, F.C. Análise de frutos e nozes de cultivares de nogueira-macadâmia. Ciência Rural, Santa Maria, v.41, n.12, p.2.0802.083, 2011.

PEREIRA-LORENZO, S.; RAMOS-CABRER, A.M.; DÍAZ-HERNÁNDEZ, M.B.; CIORDIAARA, M.; RÍOS-MESA, D. Chemical composition of chestnut cultivars from Spain. Scientia Horticulturae, Alexandria, v.107, p.306-314, 2006.

YAMANISHI, O.K.; SOBIERAJSKI, G.R.; BUENO, S.C.S.; POMMER, C.V. Chestnut in Brazil: researches and perspectives. Acta Horticulturae, Leuven, v.866, p.539-541, 2010. 